\title{
Empirical Capacity of mmWave WLANs
}

\author{
Candy Yiu, Student Member, IEEE, and Suresh Singh, Member, IEEE
}

\begin{abstract}
The $60 \mathrm{GHz}$ spectrum gives us the opportunity to deliver gigabit rates to users in a WLAN (Wireless Local Area Network) setting. The constrained propagation of signals at this frequency band ensures limited coverage which in turn enables the construction of very efficient STDMA (Spatial Time Division Multiple Access) schedules. In this paper we study the achievable aggregate capacity in a room when using two types of smart antenna arrays - linear and circular. Using detailed Matlab simulations, we show that with just $400 \mathrm{MHz}$ of the spectrum, aggregate data rates of $9 \mathrm{Gbps}(4.5 \mathrm{Gbps})$ can be achieved with linear (circular) arrays. We also study the energy efficiency of the communication and show that the energy/bit is as low as $0.2 \times 10^{-10}\left(0.2 \times 10^{-9}\right)$ Joules/Bit by using variable transmit powers at different parts of the room. Finally, we study the problem of coverage due to blocking of the LOS (Line Of Sight) path. To mitigate this problem we study the use of static reflectors and show that coverage in the entire room can indeed be maintained.
\end{abstract}

Index Terms-60GHz, STDMA, Capacity, WLAN.

\section{INTRODUCTION}

The 60GHz ISM band gives us an unprecedented opportunity to deliver gigabit rates to users in wireless LAN environments. The severe attenuation experienced by the signal in free space, combined with the attenuation due to absorption and diffuse reflection off most surfaces, severely limits the propagation of the signal. While this property may be viewed as a problem in terms of coverage, it also gives us an exciting opportunity to maximize spatial reuse and thus achieve very high data rates. In this paper, we study the achievable data rates for simple STDMA algorithms in indoor environments. Assuming that the users as well as a ceiling mounted access point have smart antennas, we show that aggregate data rates of over 9Gbps can be achieved when using linear antenna arrays and $4.5 \mathrm{Gbps}$ when using circular antenna arrays while utilizing only $400 \mathrm{MHz}$ of bandwidth. The energy per bit is less than $0.2 \times 10^{-10} \mathrm{~J} / \mathrm{Bit}$ for the linear antenna case and $0.2 \times 10^{-9} \mathrm{~J} / \mathrm{Bit}$ for the circular antenna for a BER of $10^{-6}$.

The approach we follow is to first pre-partition the room into regions or "soft cells" and then allocate spectrum to users based on their location vis-à-vis these regions. The reason to use static regions is two fold - first, it simplifies channel allocation in actual deployed systems and, second, given the ray-like propagation of $60 \mathrm{GHz}$ signals, we can develop simple STDMA algorithms within this framework. We first examine the nature of the regions formed when using two types of antenna arrays - linear and circular. Then, for each case, we study the performance of simple STDMA algorithms. Since we are interested in capacity, we make no assumptions on number

Both authors are with the Department of Computer Science, Portland State University, Portland, OR, 97207 USA e-mail: (see http://www.cs.pdx.edu/singh). This work was funded by the NSF under grant number: CNS-0722008. of users. Rather, we assume that users are located randomly uniformly anywhere in the room and thus we compute the maximum achievable data rate in the room when all locations have users. An interesting result we get is that the linear antenna array generally has higher capacity as compared with the circular antenna array consisting of the same number of elements.

The remainder of this paper is organized as follows. The next section presents a brief overview of related research on the millimeter band. Section III presents the system model we use in the study. Following that, in section IV, we use the unique propagation properties of this frequency band to create "soft cells" that form the basis for spectrum allocation. Section $\mathrm{V}$ discusses how transmit power is determined and interference mitigation. Section VI describes the problem of creating STDMA schedules. We describe the algorithms used for linear arrays in section VI-A and for circular arrays in section VI-B. The problem with the $60 \mathrm{GHz}$ band is the ease with which links can break - either due to user mobility or other static obstructions. We explore the use of reflectors to mitigate this problem in section VII. Finally, we conclude in section VIII.

\section{RELATED WORK}

$60 \mathrm{GHz}$ is well-absorbed by oxygen in the atmosphere and by several materials [14], [6], [8], [5], [13] leading to the absence of a rich multipath environment. Thus, LOS paths are the predominant signal path and due to the severe attenuation of reflected signals, this part of the spectrum is well-suited to highly efficient spatial frequency reuse. Article [3] reports on propagation measurements at $60 \mathrm{GHz}$ and $2.5 \mathrm{GHz}$. They note that large-scale propagation for $60 \mathrm{GHz}$ can be modeled as free space (a measured path loss exponent of 2.1 in buildings) as was also noted by [2]. Other results of these and other measurement studies [18] note the small RMS delay spread for $60 \mathrm{GHz}$ indicating little multipath. Article [19] presents a detailed measurement study of $60 \mathrm{GHz}$ propagation in indoor environments with particular attention to multipath. One interesting result is that the strongest reflected components off common polished surfaces in offices are at least $10 \mathrm{~dB}$ below the LOS component. Second-order and higher-order reflections are highly attenuated and negligible. Penetration loss through walls in the building are very high with many examples of over $35 \mathrm{~dB}$.

In [17] a MAC design for a multihop $60 \mathrm{GHz}$ WPAN is presented. Every node uses highly directional antennas and high transmit power to maintain a network-wide rate of 2Gbps. However, the paper does not consider the attenuation due to different materials that may obstruct the signal path (including the human body). We finally note that STDMA with 
smart antennas for indoor environments has been well studied. However, unlike previous models [16], [1], [12], [11], [10] that rely on multipath and RAKE receivers, in our case we only use the LOS path and null the NLOS paths. Therefore, the channel allocation problem is very different as we show in the remainder of this paper.

The capacity problem for $60 \mathrm{GHz}$ has received attention in the past couple of years. For instance, in [4] the authors study the problem for the case when using an antenna array. They study the capacity achieved when using SIMO, MISO, and MIMO configurations. They observe a capacity of $4.3 \mathrm{~b} / \mathrm{s} / \mathrm{Hz}$ in $90 \%$ of the cases. In [15] the author studies the impact of antenna directionality on capacity under two channel models. The key results are that using a smart antenna is necessary (rather than a fixed directional antenna) and it is necessary to have optimal pointing. An aggregate capacity of over 30 Gbps is achieved for receive antenna gain of $25 \mathrm{dBi}$. Our work differs from these papers in the following ways. First, we present algorithms for determining transmission schedules and algorithms for creating spatial channels. Next, we exploit excess antenna elements present at the AP to form multiple nulls in some set of directions to reduce interference to negligible levels. Finally, we study the problem of link breakage and how to repair these links via reflectors. In other words, while the previous works provide good bounds, our paper approaches the problem for the point of view of architecting the system and then evaluating the achievable capacity.

\section{SySTEM MODEL}

We assume a $10 \mathrm{mx} 10 \mathrm{mx} 3 \mathrm{~m}$ room for the purposes of our simulations. As will be evident, given the spatial reuse of the channel, the results can be simply extrapolated to larger room sizes as well. The AP (Access Point) is assumed to be in the center of the room in the ceiling. We study the performance of two array topologies - linear and circular. In the case of the linear antenna array, the array axis is parallel to the x-axis. For the purposes of our study, we use detailed propagation models developed in Matlab and based on ray tracing. We use the attenuation data from [19], [18], [3] to model the attenuation suffered by reflection and penetration.

The AP and users are both assumed to be equipped with smart antennas consisting of $M$ elements. In the case of the $\mathrm{AP}$, we assume that there are $k$ beamforming modules present. Thus, the AP can simultaneously support $k$ communication links (of course, each module has its own RF chain). The array factor (AF) used for the linear array is [9],

$$
A F=\sum_{i=1}^{M} e^{j(i-1) k d\left(\sin \theta-\sin \theta_{0}\right)}
$$

where $k=2 \pi / \lambda$ and $d$ is the antenna element spacing. $\theta_{0}$ is the angle at which we are forming the main beam and $\theta$ is the angle at which we are computing the array factor. The AF for the circular array when beamforming in a direction $\left(\phi_{0}, \theta_{0}\right)$ is [9],

$$
A F=\sum_{i=1}^{M} e^{-j\left(k a\left(\sin \theta \cos \left(\phi-\phi_{i}\right)-\sin \theta_{0} \cos \left(\phi_{0}-\phi_{i}\right)\right)\right)}
$$

where we again measure the angles as shown in Figure 2. Gain for both cases is computed as,

$$
E \frac{4 \pi U(\theta, \phi)}{\int_{0}^{2 \pi} \int_{0}^{\pi} U(\theta, \phi) \sin \theta d \theta d \phi}
$$

where $E$ is the efficiency (that we assume is 1 in this paper). $\theta$ and $\phi$ are measured as shown in Figure 2 and $U$ is the radiation intensity derived from the $\mathrm{AF}$ [9].

The STDMA algorithms we develop rely on effective nulling of reflected signals. Let us assume that we are beamforming at angle $\theta_{0}$ and nulling angles $\theta_{1}, \cdots, \theta_{p}$. We can write the following set of linear equations for the linear antenna array:

$$
\begin{aligned}
\sum_{i=1}^{M} w_{i} e^{j(i-1) k d \sin \theta_{0}} & =1 \\
\sum_{i=1}^{M} w_{i} e^{j(i-1) k d \sin \theta_{1}} & =0 \\
& \cdot \\
\sum_{i=1}^{M} w_{i} e^{j(i-1) k d \sin \theta_{p}} & \cdot \\
& =0
\end{aligned}
$$

Here, each equation describes the desired output of the antenna for the different directions. By solving these equations for $w_{i}$, we can beamform towards $\theta_{0}$ and null the remaining $p$ angles. In matrix form, we can write the above set of equations as $w^{H} \cdot A=[1,0, \cdots, 0]^{T}$ where ${ }^{H}$ denotes the Hermitian and $A$ is the matrix of array factors. If $p=M-1$, the above system can be solved in matrix form as:

$$
w^{H}=[1,0, \cdots, 0]^{T} A^{-1}
$$

Otherwise, if $p<M-1$ we use an approximation [7] where we make the matrix $A$ square by adding noise terms. The weights are then found as:

$$
w^{H}=[1,0, \cdots, 0]^{T} A^{H}\left(A A^{H}+\sigma^{2} I\right)^{-1}
$$

where $\sigma^{2}$ is the noise variance.

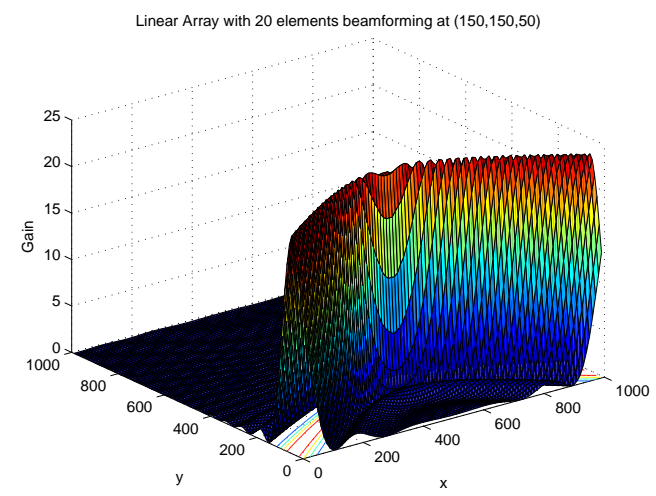

Fig. 1. Gain with 20 -element linear array pointing to $(1.5 \mathrm{~m}, 1.5 \mathrm{~m}, 0.5 \mathrm{~m})$.

\section{Creating Cells or Regions}

The key idea that we use in this work is that of prepartitioning the room into non-overlapping regions and then using these regions as the basis for channel allocation. Let us first consider the linear antenna array case. Figure 1 shows the gain from the AP to the user (beamforming at the AP) 


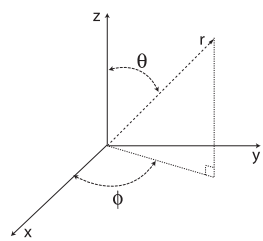

Fig. 2. 3D angle convention used.

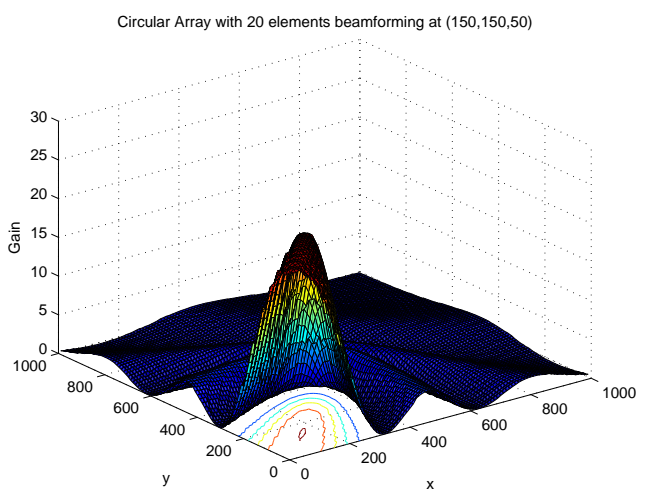

Fig. 3. Gain with a 20 -element circular array pointing to $(1.5 \mathrm{~m}, 1.5 \mathrm{~m}, 0.5 \mathrm{~m})$.

who is located at $(1.5 \mathrm{~m}, 1.5 \mathrm{~m}, 0.5 \mathrm{~m})$. As is evident, the rotational symmetry (about the array axis) causes the region of highest gain to be spread in a narrow strip across the floor of the room (when viewed on the $x-y$ plane). Indeed, if we sweep the beam across all the angles, we will see this strip move in the direction of the $y$-axis. This behavior gives us a natural way to think about constructing multiple spatial channels simultaneously. For our sample room, we partition the room into 21 regions as illustrated in Figure 4. The gain drops by $3 \mathrm{~dB}$ from the center of a region to its edge as shown in Figure 5. Of course, if the room is larger, there will be more regions and, similarly, we can change the definition of the region's boundary.

Given a partitioning of the room as illustrated it is easy to see how one would design STDMA algorithms. For instance, given two modules, we can form one beam at some region $i$ and the other at region $j$ such that the interference between these two beams is low enough to maximize throughput. To cover the entire room, we now form a STDMA schedule where, in each slot of the schedule, two regions get coverage simultaneously. Extending this idea to the case when the AP has $k$ modules is trivial.

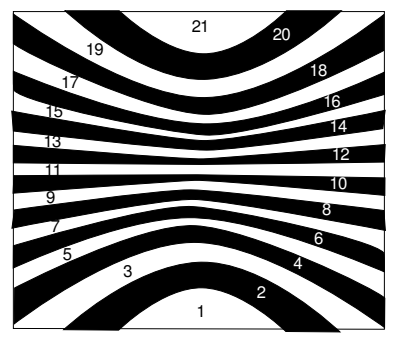

Fig. 4. The 21 regions formed with a linear array.

Figure 3 shows the gain from the AP to the user located

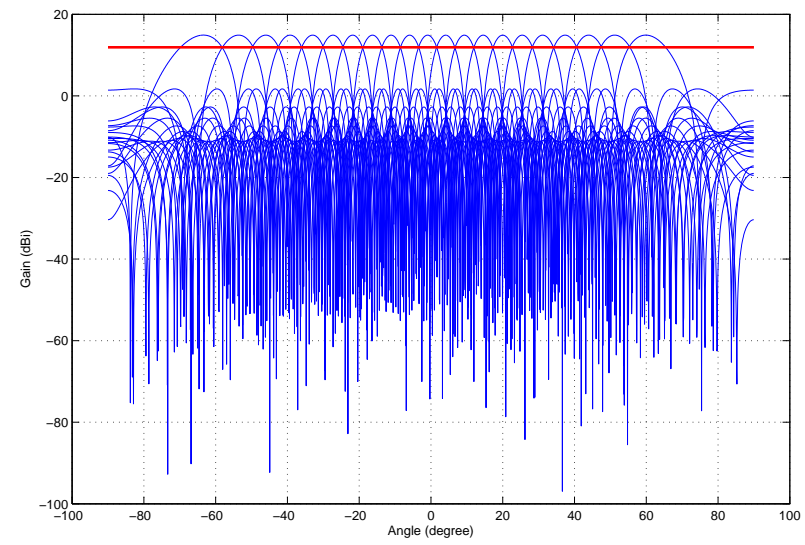

Fig. 5. The gain seen at the 21 regions formed with a linear array.

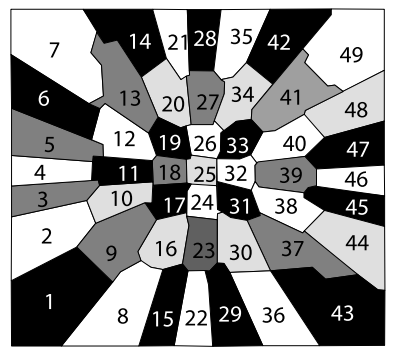

Fig. 6. The 49 regions formed with a circular array.

at $(1.5 \mathrm{~m}, 1.5 \mathrm{~m}, 0.5 \mathrm{~m})$ when using a circular antenna array. Following the same process as for the linear antenna case, we can define regions in order to cover the entire room. Again, gain at the region boundary is $3 \mathrm{~dB}$ below the gain at the center of the region. Figure 6 shows the 49 regions formed with the circular array. As in the linear array case, we can now construct STDMA schedules using this structure.

\section{Transmit Power Selection and Interference Mitigation}

Before we study the use of the region idea for creating STDMA schedules, we need to consider the simultaneous challenge of conserving energy and countering LoS interference and first-order reflections. The distance between the AP and various parts of the room varies from $3 \mathrm{~m}$ (directly below the AP) to $7.8 \mathrm{~m}$ at the very corner of the room. Thus, the path loss at the corner of the room is $8 \mathrm{~dB}$ below that in the center of the room. In other words, we can safely use less transmit power when transmitting to the center of the room than to the edges.

In order to determine the needed transmit power at different parts of the room, we perform a simple link budget calculation. Following [8], we use a $400 \mathrm{MHz}$ channel and consider uncoded 64-QAM as the target. Using standard techniques, we calculate a $\mathrm{SNR}_{\min }$ requirement of $23.4 \mathrm{~dB}$ in order to achieve Gbps data rate with a BER of $10^{-6}$. Let us assume a receiver 


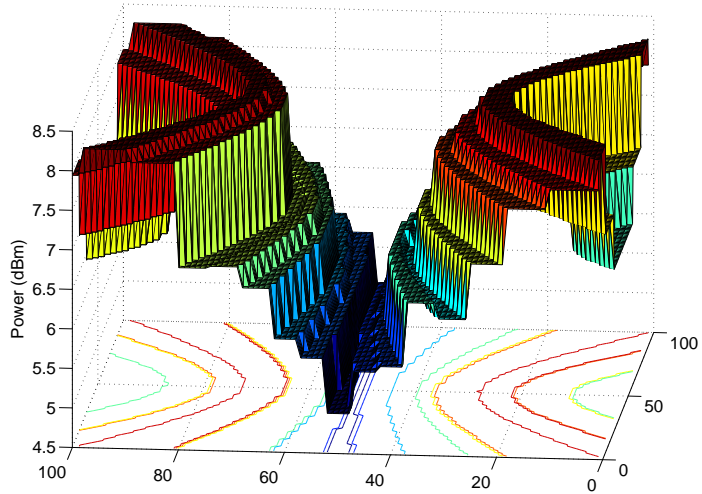

Fig. 7. Transmit power used in different regions (Linear Array).

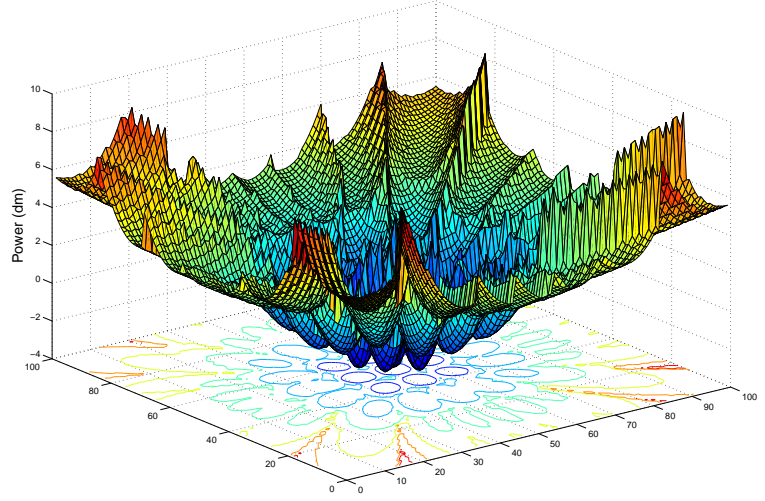

Fig. 8. Transmit power used in different regions (Circular Array).

noise figure of $10 \mathrm{~dB}$. Then we get a sensitivity of,

$$
\begin{aligned}
S= & -174 \mathrm{dBm} / \mathrm{Hz}+10 \log _{10}(400 \mathrm{MHz}) \\
& +10 d B+23.4 d B=-54.57 d B m
\end{aligned}
$$

Let $G_{1}(i)$ and $G_{2}(i)$ be the AP and user antenna gains respectively when pointed to (or from) region $i$. Then, the minimum required transmit power for region $i$ is,

$$
\begin{array}{r}
P_{t x}(i)=\max _{d} P_{t x}(d, i)=\max _{d}\left(S-G_{1}(i)-G_{2}(i)\right. \\
\text {-Path Gain }(d, i))
\end{array}
$$

$P_{t x}(d, i)$ is the transmit power required in region $i$ when the user is distance $d$ away (since regions are long strips, $d$ varies).

Assuming that the antennas have 20 elements each, Figure 7 plots $P_{t x}+3 d B$ for the linear antenna array. As is clear, significant power savings are possible if we match the transmit power to the user location. Figure 8 plots the transmit power for the circular antenna case as well. Again, we see a big difference in the required transmit power at different locations. These are the power values used in all experiments.

In practice, there will be interference from other simultaneous transmissions which will lower the SINR achieved. The interference comes about from LoS transmissions to other regions as well as from first-order reflections off the floor and walls. In order to counter the LoS interference, we use two techniques - the transmit power we use is $3 \mathrm{~dB}$ above the $P_{t x}$ value and we use $1 / 2$ or $3 / 4$ convolution coding (constraint length 8) that will give us a gain of between $3-7 \mathrm{~dB}$. For the first-order reflections, it turns out that the total interference from all reflections is very small and can be ignored, as discussed next.

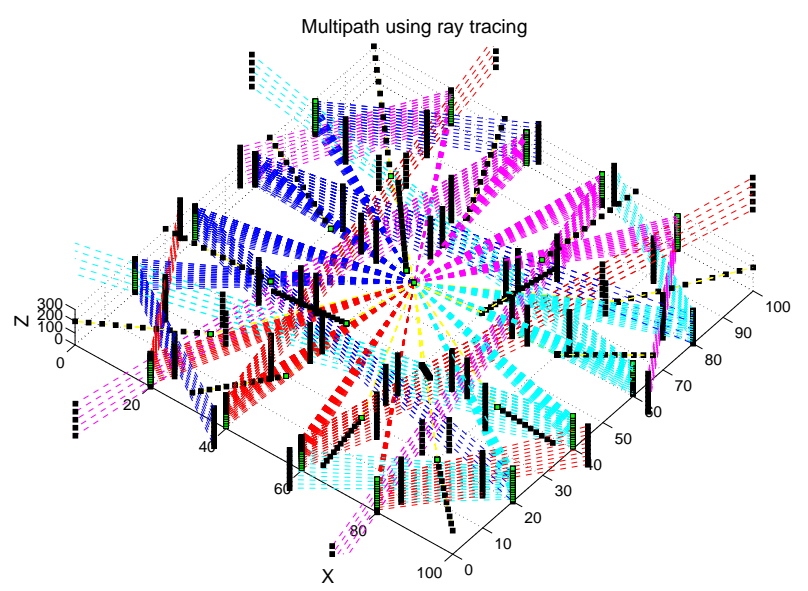

Fig. 9. First-order reflection paths.

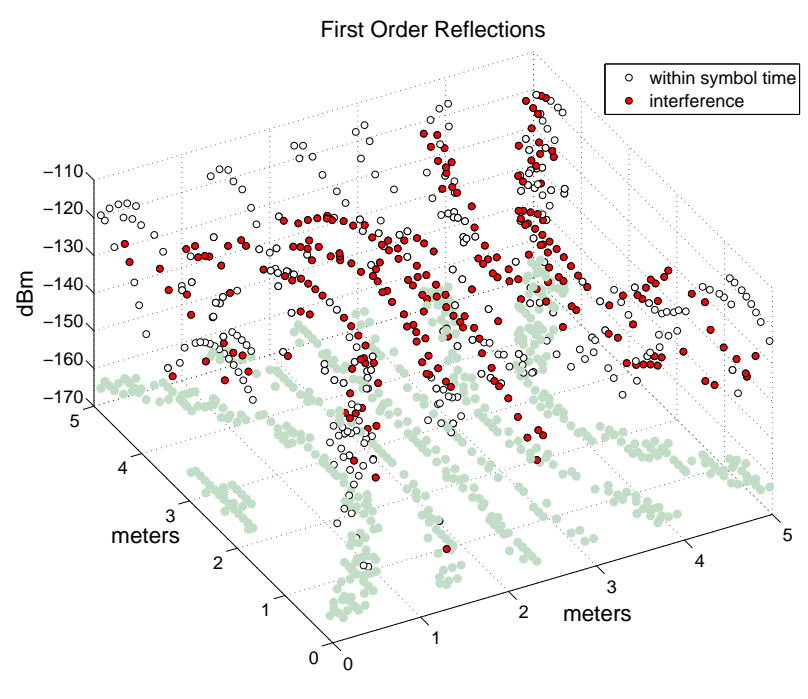

Fig. 10. Sum of all first-order interference when using seven regions.

To measure the power level of first-order reflections, we use ray tracing where for each point in the room, we sum up the interference power from all first-order reflections. Some reflections arrive within the symbol time $(2.5 \mathrm{~ns}$ for $400 \mathrm{MHz}$ bandwidth) while others arrive late. Figure 9 illustrates the set of first-order reflection paths. The access point is in the center of the room on the ceiling. Assuming that seven of the 21 regions for the linear array case are simultaneously scheduled to transmit and receive in the same channel, we plot the interference from first-order reflections in Figure 10 (we assume the reflections suffer a $30 \mathrm{~dB}$ attenuation). The 


\begin{tabular}{|l|l|}
\hline Room dimensions & $10 \mathrm{~m} \times 10 \mathrm{~m} \times 3 \mathrm{~m}$ \\
AP location & Center of ceiling \\
Transmit power & Figures 7, 8 \\
Bandwidth & $400 \mathrm{MHz}$ \\
Modulation & Table II \\
No. antenna elements AP and user & $M=20$ \\
No. Modules $k$ at AP & $1-21$ (Linear) \\
& $1-49$ (Circular) \\
\hline
\end{tabular}

TABLE I

EXPERIMENTAL PARAMETERS.

projection on the floor shows the seven regions selected while the points show the total interference and total signal energy arriving within one symbol time. First, note that the total energy is well below $-110 \mathrm{dBm}$ in all cases which means that interference from first-order reflections is negligible. Second, note that points in the center of the room display more interference - this makes sense since signal paths are longer and therefore arrive after $2.5 \mathrm{~ns}$.

\section{Constructing STDMA Schedules}

We now return to the problem of using the region concept to develop algorithms for finding STDMA schedules. It is convenient to think of the approach as a 2-step process. First, we create a schedule to cover the entire room regardless of user presence. This will give us the achievable capacity of the WLAN. Second, in practical circumstances, when users are only present in some subset of regions, we can modify the schedule (shorten it) to only provide coverage where needed.

To measure achievable capacity of a given STDMA schedule, we compute the SINR at each point in each region and obtain the data rate for each point. Then, assuming that users may be placed randomly uniformly in the region, we calculate the average data rate achieved in that region. The capacity is then the total data rate achieved in the schedule divided by the number of slots in the schedule. Let the STDMA schedule have $s$ slots and in each slot one or more regions are active simultaneously. Let $r_{i}$ be the average data rate achieved in region $i$ (on average) in its scheduled slot. The achievable capacity is then,

$$
\frac{\sum_{i=1}^{R} r_{i}}{s}
$$

where $R=21$ for the linear array and 49 for the circular array. In order to compute the average energy per bit, we do the following computation:

$$
\sum_{i=1}^{R}\left(\frac{P_{t x}(i)}{r_{i}} \frac{r_{i}}{\sum_{i=1}^{R} r_{i}}\right)=\frac{\sum_{i=1}^{R} P_{t x}(i)}{\sum_{i=1}^{R} r_{i}}
$$

The expression on the left is simply the energy per bit in region $i$ normalized by the data rate in that region. $P_{t x}(i)$ is the transmit power used in region $i$ (as in Figure 7).

\section{A. STDMA for the Linear Array}

The problem of creating a STDMA schedule can be stated simply as follows: given $k$ beamforming modules at the AP, create a STDMA schedule that maximizes achievable capacity. In this paper, we explore the relationship between achievable

\begin{tabular}{|c|c|c|c|}
\hline Modulation & Uncoded & 3/4 Conv. & 1/2 Conv. \\
\hline 64-QAM & 18.78 & 14.08 & 12.23 \\
16-QAM & 14.40 & 9.7 & 7.85 \\
QPSK & 10.52 & 5.82 & 3.97 \\
BPSK & 10.52 & 5.82 & 3.97 \\
\hline
\end{tabular}

TABLE II

Minimum $E_{b} / N_{0}$ REQUIREMENTS (BER $10^{-6}$ ).

capacity and $k$. Thus, we vary $k$ from 1 to 21 for the linear array and between 1 and 49 for the circular array.

For $k$ modules, we can simultaneously form $k$ beams. To ensure that interference between beams is minimized, we maximize the angular separation between the beams. For example, if $k=3$, we have the following schedule: $\{(1,8,15)$, $(2,9,16),(3,10,17),(4,11,18),(5,12,19),(6,13,20),(7,14,21)\}$. Thus we use 7 slots and in each we simultaneously have 3 links. The schedule for $k=8$ is: $\{(1,4,7,10,13,16,19,21)$, $(2,5,8,11,14,17,20),(1,3,6,9,12,15,18,21)\}$. As we can see, when the number of regions is not perfectly divisible by $k$, the separation of regions in the same time slot is not uniform. Given $r$ regions and $k$ modules, the separation between regions in the same time slot is either $\left\lfloor\frac{r}{k}\right\rfloor$ or $\left\lceil\frac{r}{k}\right\rceil$.

In our experiments, we also use the nulling capability of smart antennas. Thus, given $k$ modules, we form $k$ simultaneous beams - each module beamforms in one direction and nulls the remaining $k-1$ directions. For instance, if $k=3$ and regions $(1,8,15)$ are active simultaneously, the module that beamforms towards region 1 also nulls the center point of regions 8 and 15, and so on. An interesting observation we can make is that since our antennas have $M=20$ elements, we can potentially form 19 nulls per module. Thus, if $k<M$ and we form $k-1$ nulls as described, we still have the capability to form an additional $M-k$ nulls per module. The question is where to form the additional nulls, and whether this is a useful thing to do.

We research this question in some detail. Consider Figure 11 where we beamform towards region 1 and null the center of region 11 and we only use $k=2$ beamforming modules. If we look at the gain at areas close to the null, we see a steep increase in gain - the center of region 11 is at $-48 \mathrm{dBi}$ while the edges are at $-30 \mathrm{dBi}$. This means that even if we null the center of any region from Figure 4 , it is likely that there will be significant gain as we go to the boundaries of that region, which will result in more interference. This observation means that given the opportunity to form additional nulls, we should form more than one null per region at different angular locations such that interference in that region is reduced overall. In Figure 12 we plot the case when we form $M-1$ nulls in region 11 (each null is incrementally located a small fraction of a radian off center) while beamforming to region 1 and vice versa. We see that the interference from beamforming at region 1 towards region 11 is now well below $-100 \mathrm{dBi}$ across the entire span of region 11 (and vice versa). This clearly indicates that forming multiple nulls within a region is a beneficial thing to do.

We compared the cases when modules only form $k-1$ nulls and when modules form $M-1$ nulls. The algorithm we used 


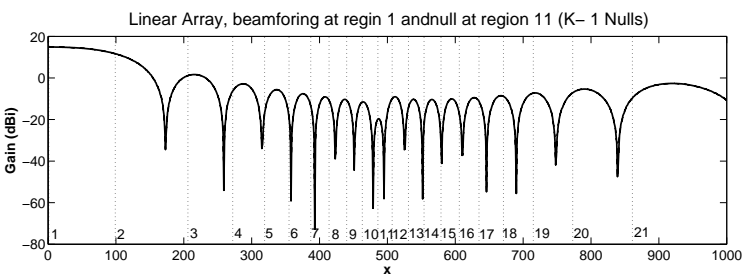

Fig. 11. Beamform to region 1, place a single null in region 11 .

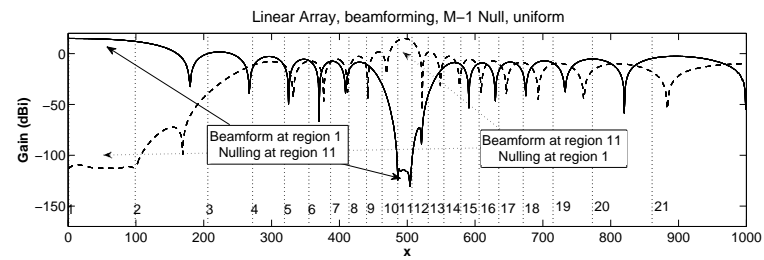

Fig. 12. Forming 19 nulls in each region.

to allocate the additional $M-k$ nulls is as follows. For each of the $k-1$ nulling directions, we allocate $\left\lfloor\frac{M-k}{k-1}\right\rfloor$ additional nulls. These nulls are then formed at angles $\pm \epsilon, \pm 2 \epsilon, \cdots$ about the center of the region until we form all the allocated nulls. The performance of the algorithm for different values of $\epsilon$ are somewhat different and in our plots we report on only two extreme values, $\epsilon=0.0005,0.03$ radians. Another method we consider is called uniform where we first find the beamwidth of a region (i.e., the $3 \mathrm{~dB}$ boundary) and then distribute the $\left\lfloor\frac{M-1}{k-1}\right\rfloor$ nulls equally spaced (in an angular sense) in the region. Note that in all experiments the user also beamforms towards the AP using an identical antenna array. However, the user does not form nulls since interference from uplink transmissions from other users is negligible.

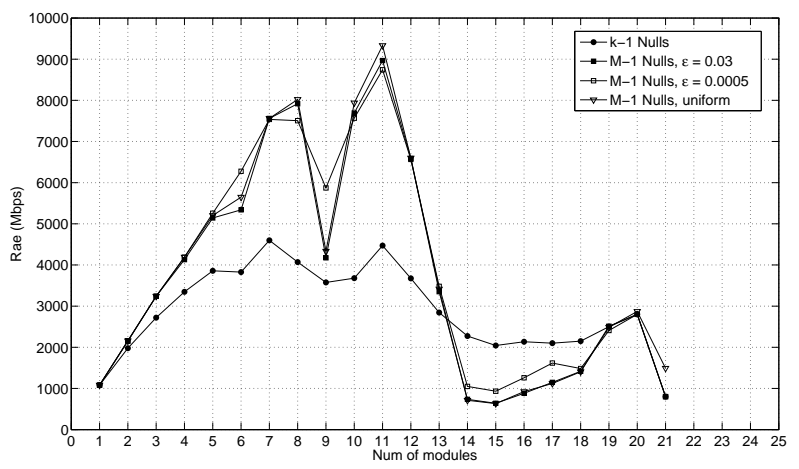

Fig. 13. Average aggregate capacity for the linear array case.

Figure 13 plots the capacity achieved as a function of the number of modules at the AP. The maximum capacity of over $9 \mathrm{Gbps}$ is achieved when using 11 modules and when we form $M-1$ nulls per module. Interestingly, if we only form $k-1$ nulls, the the capacity is less than half! The reason that forming more nulls is better is simply that we reduce interference levels in the region as a whole from other beams. Since the average is computed by assuming a uniform random user placement over the entire region, more nulls provide a greater overall benefit. Increasing the number of modules beyond 13 appears to be detrimental for the case when forming $M-1$ nulls. In Figure 14 we plot the signal levels at each region when using $k=14$ modules and beamforming at region 1 . There is one plot for the case when forming $M-1$ nulls using the uniform algorithm (i.e., there are 14 regions active simultaneously so when beamforming to region 1 , we form one null in each of the 13 other regions and an additional null in regions 2, 3, $4,5,6,7)$ and for the case when forming $k-1$ nulls. As is clear, the desired signal in region 1 is much higher when we only form $k-1$ nulls. The reason is that as we form more and more nulls, the main beam flattens out and spreads more. Therefore, there is an optimal number of nulls one can form that trades off the benefits of reducing interference with the cost of reducing the desired signal strength.

Finally, there is an interesting drop in throughput when using 9 modules. Consider the 8, 9, and 10 module cases. In each case, the schedule used is 3 time slots long (ceiling of the number of regions/number of modules). The total number of regions that get the channel during the three slots is 24,27 , and 30 respectively. Therefore we expect the throughput for 10 to be greater than that for 9 , and for 9 to be greater than that for 8 . However, the throughput for 9 modules is less than for 8 modules. The reason is that the separation of simultaneously active regions when using 8 modules is 3 whereas it is 2.14 for 9 modules and 1.97 for 10 modules. The interference is greater when the separation is 2 rather than when it is 3 which is why we get higher throughput with 8 modules than with 9 . The difference in separation between 9 and 10 modules is small but we have more transmissions with 10 modules which has the net effect of increasing throughput

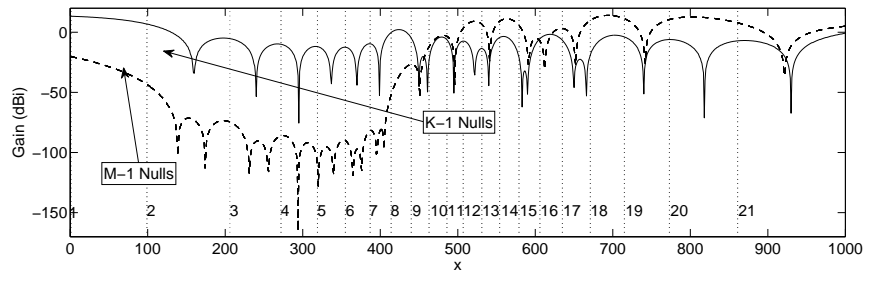

Fig. 14. Comparison of the desired signal levels when using 14 modules.

Figure 15 plots the energy/bit as a function of the number of modules used. Consider first the case when only one module is being used. This essentially means that the data rate achieved is $1 \mathrm{Gbps}$ but only one region is active at a time. Therefore, the energy/bit is also small. The energy per bit remains small as we increase the number of modules since the data rate also grows - i.e., we are utilizing the available bandwidth efficiently. However, after 12 modules, the energy/bit increases sharply for the $M-1$ uniform case because the data rate falls (increased interference) but we use more power since more regions are active simultaneously. Putting Figures 13 and 15 together, it is clear that the sweet spot that maximizes aggregate capacity and minimizes energy is when using 11 modules.

\section{B. STDMA for the Circular Array}

Creating a STDMA schedule for the circular array case is more challenging due to the more irregular structure of the 


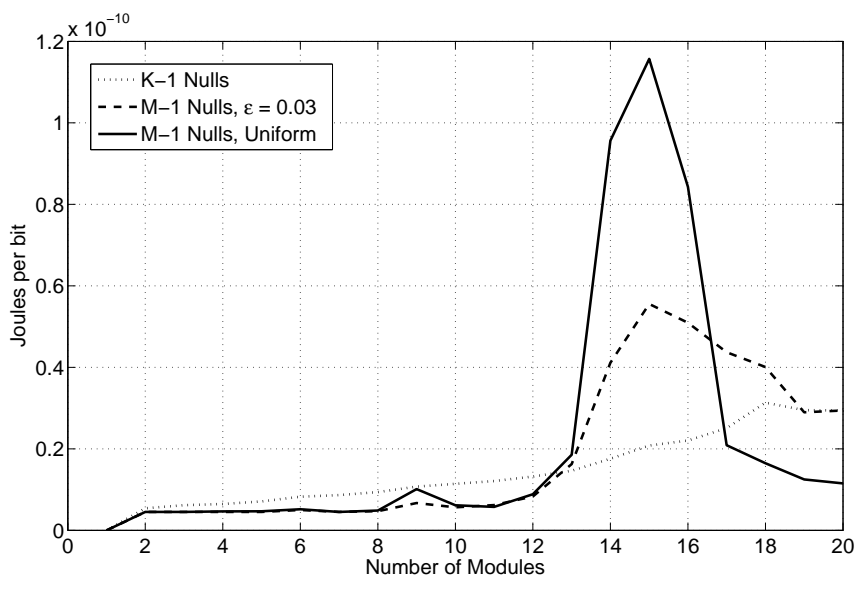

Fig. 15. Energy/bit for the linear array.

regions. However, experimentally we observed that a simple algorithm modelled after the algorithm for the linear array, performs very close to optimal. The algorithm we use is as follows. Given $k$ modules, we compute $d=\left\lfloor\frac{49}{k}\right\rfloor$. The schedule then is: $\{(1, d+1,2 d+1, \cdots,(k-1) d+1),(2$, $d+2,2 d+2, \cdots,(k-1) d+2), \cdots,(\mathrm{d}, 2 d, 3 d, \cdots, k d)\}$. If the number of regions is not perfectly divisible by $k$ then we will need an additional time slot to accommodate the remaining regions.

The problem of forming nulls is similar to the discussion we had previously. Thus, we consider the case when we form only $k-1$ nulls given $k$ modules and when we form $M-1$ nulls. However, the algorithms for forming $\left\lfloor\frac{M-1}{k-1}\right\rfloor$ nulls per region are different. The first algorithm, called $\epsilon$-walk is illustrated in Figure 16. The idea is that we walk along a grid of side $\epsilon$ around the center of a region and use the vertices as locations for the additional nulls. The second algorithm is illustrated in Figure 17. Here, we take the region to be nulled and uniformly distribute $\left\lfloor\frac{M-1}{k-1}\right\rfloor$ nulls within it. The difference between the $\epsilon$-walk and the uniform case is that in the $\epsilon$-walk algorithm, the nulls will tend to be clustered about the center of the region while the nulls are uniformly distributed about a region in the other case.

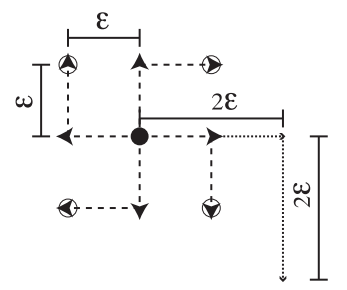

Fig. 16. Epsilon walk algorithm.

Figure 18 plots the achievable capacity as a function of number of modules for the circular array. A maximum of $4.5 \mathrm{Gbps}$ is achieved when using either 5 or 10 modules and forming $M-1$ nulls. The explanation for the max at 5 modules is as follows. The schedule created is $\{(1,10,19,28,37)$, $(2,11,20,29,38), \cdots\}$ and so on. As we can see, the regions are well separated from one another and therefore interference

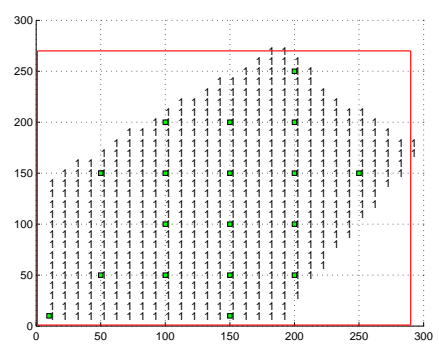

Fig. 17. Uniform placement of 16 nulls (the 'o' indicates the location of the null while the ' 1 ' is used for shading the entire region).

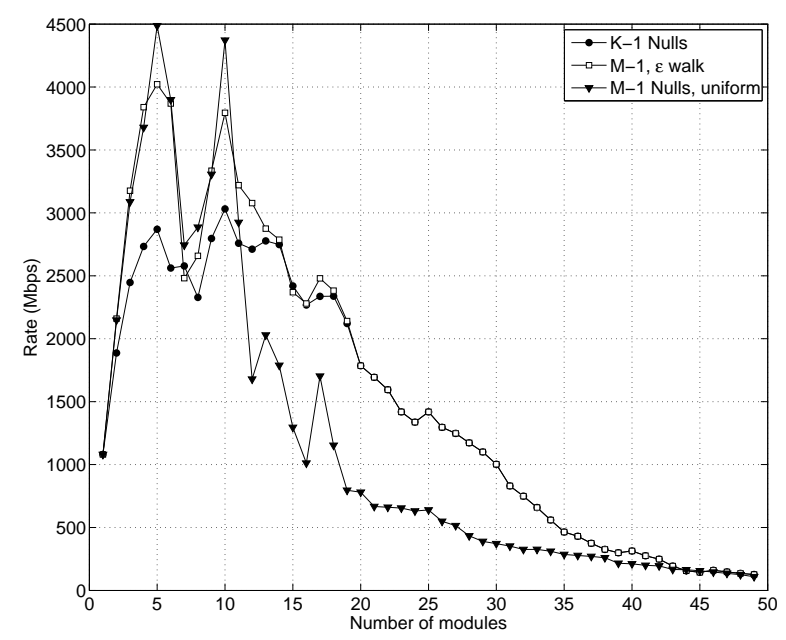

Fig. 18. Average aggregate capacity for the circular array case.

is low. Compare this with the schedule using 6 modules: \{ $(1,9,17,25,33,41), \cdots\}$. If we look at Figure 6 , we note that the regions active simultaneously with 6 modules are all right next to one another - this causes more interference among them. Furthermore, nulling in this case causes the main beam in each region to shift substantially. In the case of 5 modules, the regions are well separated and we maximize SINR in each region. The second maximum is achieved at 10 modules. In this case, the schedule is: $\{(1,5,9,13,17,21,25,29,33,37)$, $\cdots\}$. Interestingly, the regions $1,9,17,25,33$ are next to each other and thus, following the same argument as in the 6 module case, we would expect poor throughput. However, there are two additional factors that come into play. First, the other five regions are well-separated and they will show good throughput. And second, with 10 modules the schedule length $s$ is half which gives us a greater overall capacity. Of course, as the number of modules increases further, the number of active regions that are neighbors also increases and the benefits of a shorter schedule are lost.

Figure 19 plots the energy/bit when using the circular array. Following the explanation given for Figure 15, we see that initially as the number of modules increase, the energy/bit remains unchanged - we are essentially exploiting available capacity efficiently. However, after the number of modules exceeds 15 , the energy/bit starts rising sharply. This 


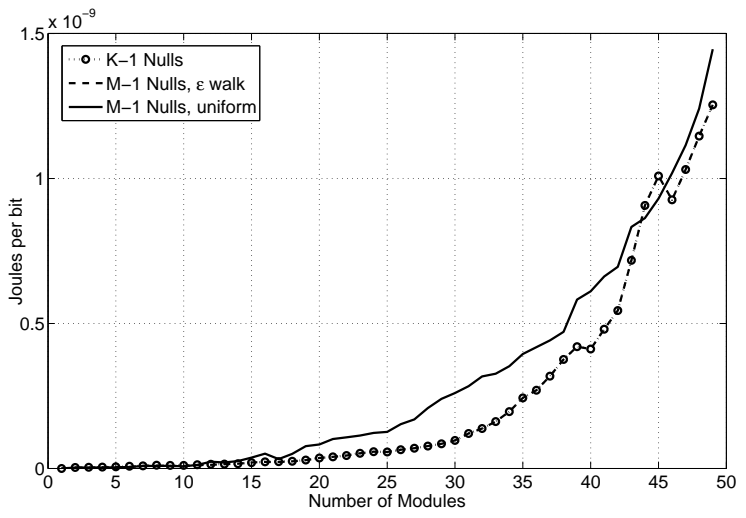

Fig. 19. Energy/bit for the circular array.

is because of decreased capacity due to increased interference. Interestingly, the $M-1$ uniform algorithm shows the fastest rise in energy. The reason for this is that with the nulls spaced out uniformly over regions, the main beam shifts and flattens more - causing increased interference. In contrast, the $\epsilon$-walk algorithm concentrates the nulls about the region's center and thus results in less shifting. The best performance can be seen to occur at 10 modules when we get the highest rate and lowest energy/bit.

Finally, observe that there is an order of magnitude difference in the energy/bit between the linear array and the circular array. The explanation for this is two fold. First, the aggregate capacity achieved for the linear case is double that for the circular case. And second, the schedule lengths for the circular case are much longer since there are 49 regions as opposed to 21 regions that need to be covered.

\section{USE OF REFLECTORS}

The $60 \mathrm{GHz}$ spectrum is susceptible to oxygen absorption and higher attenuation when encountering different materials in its path. The implications of this poor signal propagation for WLAN design means that the connectivity of users may be poor. Thus, a user may well be blocked by a wall or partition from the AP resulting in poor signal quality resulting in very low data. Similarly, a user's link may be intermittently broken by the movement of other people in the room. Finally, as a user with an open connection moves about the room, the link may again be subject to obstructions. The challenge is how to mitigate these various types of connectivity problems.

The approach we study in this paper is to use strategically placed reflectors to provide alternate paths for the signal to reach such disadvantaged users. Materials such as wire mesh glass [19] have excellent reflective properties at $60 \mathrm{GHz}$ and only attenuate the signal by $3 \mathrm{~dB}$ for most angles of incidence. Thus, by placing sections of this material strategically on the walls of the room, the AP can provide better coverage.

Let us examine the problem of integrating reflectors into the STDMA schedules developed for the linear and circular antenna arrays respectively. In the case of a linear array, say we were to place a reflector on one wall (assume the array axis is parallel to the $\mathrm{x}$-axis and we place the reflector on the wall parallel to the $\mathrm{x}-\mathrm{z}$ plane). This has the effect of reflecting some regions back into the room. Consider Figure 20 where we assume that two walls parallel to the $\mathrm{x}$-z plane are covered by a reflector over the bottom $1.5 \mathrm{~m}$. As we can see, each region is reflected back into the room.

In reality, we will only place small reflectors at specific locations to provide coverage in dead spots. So, for example, if we have a dead area in a part of region 4 , we can cover it with a reflection of either region $4,5,6,7$, or 8 (depending on where the dead spot is located). Let us assume that the location of the dead spot is such that it is covered by the reflection of region 5. In that case, from the point of view of constructing a STDMA schedule, we need make no changes - this part of region 4 is simply assigned to region 5 and users in the dead spot share bandwidth with the users located in region 5 .

While the scheduling issue is simple to deal with, we do have to be aware of interference that is caused by reflected signals. Thus, assume that the dead spot in region 4 is covered by the reflection of region 7 and further assume that region 4 and 7 are scheduled in the same STDMA slot. In this case, there will be some interference caused at parts of region 4 from the reflected signal of region 7 . Thus users need to place a null in the direction of the reflector. Since users are equipped with 20-element antenna arrays, this is not a problem.

We evaluate the data rate achieved with a linear array for three different cases when entire regions are a dead spot. The three cases considered are that either region 1 or region 4 or region 13 are dead spots. We used 7 modules and formed $M-$ 1 nulls from each module to maximize average throughput. For the regions covered by reflections, we use a $10 \mathrm{dBm}$ transmit power. Figure 21 plots the average data rate in each region. As expected, average throughput in all LOS regions remains high while the regions that are dead spots show lower average throughput. The reason the throughput is lower is that the signal path length is longer when going via the reflector and because of attenuation of $3 \mathrm{~dB}$ at the reflector. The throughput of region 13 is much lower than that of regions 1 and 4 because the total interference in region 13 is higher - region 13 is in the center of the room and is thus susceptible to more interference from direct as well as reflected paths. However, in general, the data rates achieved are still in the Gbps range for all cases.

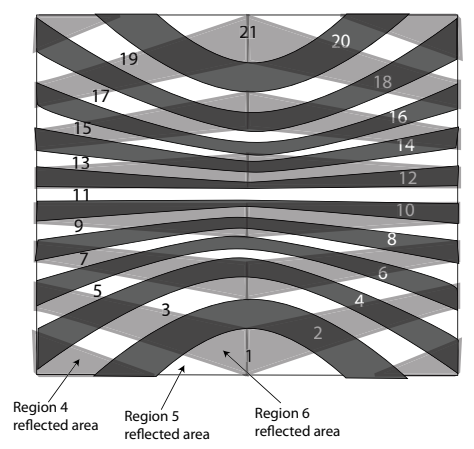

Fig. 20. Reflected regions assume two walls are reflective at the bottom $1.5 \mathrm{~m}$.

Next, let us consider the use of reflectors when the antenna array is circular. Unlike the linear case, the regions are 


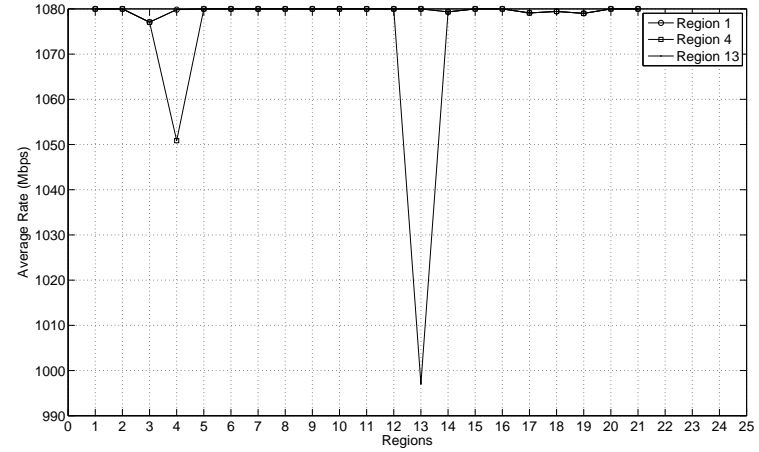

Fig. 21. Data rate for three dead spot scenarios (Linear Array).

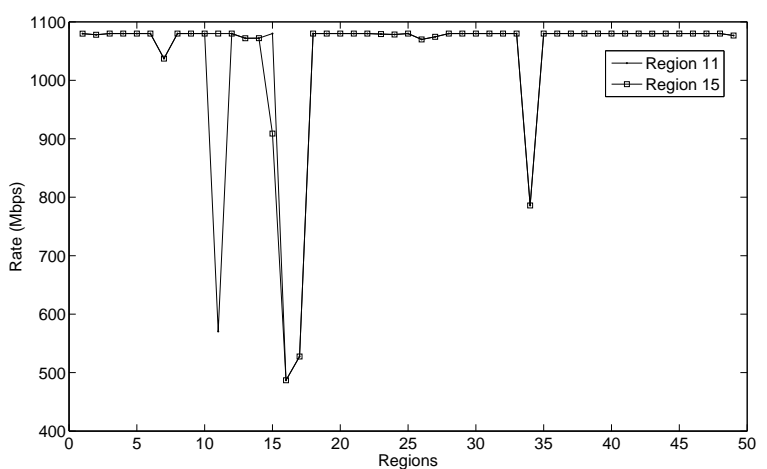

Fig. 22. Data rate for two dead spot scenarios (Circular Array).

smaller and better contained. Therefore, it is simpler to deploy reflectors and schedule transmissions. For example, if a dead spot occurs in region 15, then during the STDMA slot when region 15 is scheduled, rather than transmitting directly to region 15, the AP forms its beam at an appropriately placed reflector that covers region 15 . Thus, the schedule will not change. The drawback is the added interference at neighboring regions since the reflected beam will spread out more as it travels a longer distance (AP to reflector to region 15). Figure 22 shows the data rate for the circular array case when the regions 11 and 15 are in dead spots. The data rate at these regions when using reflectors is $600 \mathrm{Mbps}$ and $500 \mathrm{Mbps}$ respectively.

\section{CONCLUSIONS}

This paper examined the empirical capacity of an indoor WLAN operating in the $60 \mathrm{GHz}$ ISM band. Exploiting the fact that the signal propagation at this band is severely limited, we study the performance of static STDMA schedules. For the case when we use linear antenna arrays, a maximum aggregate rate of $9 \mathrm{Gbps}$ is attained for the room whereas with circular arrays this value is half. We also exploit the fact that the distance related attenuation is severe which gives us the opportunity to use variable transmit powers to different parts of the room. This improves overall capacity by reducing interference while simultaneously reducing overall power consumption. Finally, we consider the challenge of link breakage due to user mobility and presence of obstructions. To mitigate this, we use static reflectors to provide alternative coverage paths and show that this simple technique does indeed cover dead spots.

\section{REFERENCES}

[1] A. T. Alastalo and M. Kahola. Smart-antenna operation for indoor wireless local-area networks using ofdm. IEEE Transactions on Wireless Communications, 2003.

[2] S. E. Alexander and G. Pugliese. Cordless communications within buildings: Results and measurements at $900 \mathrm{mhz}$ and $60 \mathrm{ghz}$. British Telecom Technology Journal, 44(10):99 - 105, Oct. 1996.

[3] C. R. Anderson and T. S. Rappaport. In-building wideband partition loss measurements at 2.5 and $60 \mathrm{ghz}$. IEEE Trans. on Wireless Communications, 3(3):922 - 928, May 2004.

[4] A. Arvanitis, G. Anagnostou, N. Moraitis, and P. Constantinou. Capacity study of a multiple element antenna configuration in an indoor wireless channel at 60ghz. In IEEE VTC-Spring, pages 609-613, April 2007.

[5] J-P. Ebert et. al. Paving the way for gigabit networking. In Global Communications Newsletter, April 2005.

[6] G. Fettweis and R. Irmer. Wigwam: system concept development for 1 gbit/s air interface. Wireless OWrld, Rsearch Forum, 2005.

[7] L. Godara. Application of antenna arrays to mobile communications, part ii: beamforming and direction-of-arrival considerations. Proceedings of the IEEE, 85(8):1195 - 1245, Aug 1997.

[8] E. Grass, M. Piz, F. Herzel, and R. Kraemer. Draft phy proposal for 60ghz wpan: Ieee p802.15 wg on wpans, November 2005.

[9] Frank Gross. Smart Antennas for Wireless Communications. McGraw Hill, 2005.

[10] C. Hoymann. Mac layer concepts to support space division multiple access in ofdm based ieee 802.16. Wireless Personal Communications, 36(4), March 2006.

[11] I. Koutsopoulos and L. Tassiulas. Adaptive channel assignment in sdma-based wireless lans with transceiver resource limitations. Signal Processing, 2006

[12] A.M. Kuzminskiy, H.R. Karimi, D.R. Morgan, and C.B. Papadias. Downlink throughput enhancement of ieee $802.11 \mathrm{a} / \mathrm{g}$ using sdma with a multi-antenna access point. Signal Processing, 86(8), 2006.

[13] C. P. Lim, R. J. Burkholder, J. L. Volakis, and R. J. Marhefka. Propagation modeling of indoor wireless communications at $60 \mathrm{ghz}$. In IEEE Antennas and Propagation Society International Symposium, pages $2149-2152,2006$.

[14] M. Marcus and B. Pattan. Millimeter wave propagation: spectrum management implications. IEEE Microwave Magazine, June 2005.

[15] A. Seyedi. On the capacity of wideband $60 \mathrm{ghz}$ channels with antenna diversity. In IEEE GLOBECOM, pages 4532-4536, 2007.

[16] F. Shad, T. Todd, V. Kezys, and J. Litva. Dynamic slot allocation (dsa) in indoor sdma/tdma using a smart antenna basestation. IEEE/ACM Transactions on Networking, 9(1):69 - 81, Feb 2001.

[17] S. Singh, F. Ziliotto, U. Madhow, E. M. Belding, and M. J. W. Rodwell. Millimeter wave wpan: Cross-layer modeling and multi-hop architecture. In IEEE INFOCOM (Minisymposium), pages 2336 - 2340, May 2007.

[18] P. Smulders. Exploiting the $60 \mathrm{ghz}$ band for local wireless multimedia access: prospects and future directions. IEEE Communications Magazine, pages $140-147$, January 2002.

[19] H. Xu, V. kukshya, and T. Rappaport. Spatial and temporal characteristics of $60 \mathrm{ghz}$ indoor channels. IEEE Journal in Selected Areas in Communications, 20(3):620 - 630, April 2002. 